\title{
Treatment modalities in poor responder patients undergoing assisted reproductive techniques
}

\author{
Summary \\ Accomplished IVF/ICSI treatment depends on obtaining enough oocyte sample \\ and electing patients observantly. The $\mathrm{COH}$ (Controlled Overian Hyperstimulation) \\ treatment in patients with poor ovarian response is one of the most important issues of \\ IVF programmes. The ovarian response given ovarian stimulation is the most important \\ determiner in ART treatment. Although many other stimulation protocols have been \\ applied on patients with poor ovarian response, contemporarily low pregnancy rates \\ reported.
}

Keywords: poor responder, asisted reproductive technology, treatment modalities

\author{
Special Issue - 2018
}

\author{
Egemen Harun Tolunay \\ Department of Obstetrics \& Gynecology, Van Yuzuncu Yil \\ University, Turkey
}

Correspondence: Elizabeth Nista Mellott, RN, BSN, I University Dr. Cullowhee, North Carolina 28723, USA, Tel 828-227-721I, Email harunegementolunay@gmail.com

Received: April 18, 2017 | Published: November 30, 2018

\section{Introduction}

In this review, treatment and stimulation protocols in poor responder patients undergoing ART will be discussed. PubMed database was searched in August 2013 with various combination of following terms in English language: ART, poor responder, stimulation protocols, improvement pregnancy rates, ovarian response, ovarian reserve, IVF/ ICSI treatment.

\section{Definition}

Poor ovarian response was reported by Garcia et al. in 1983, first. Poor responder cases consitute $9-24 \%$ of ART cycles. The rate is reported as $50 \%$ in women over 40 years old. ${ }^{2} \mathrm{FSH}$ begins increasing before 13 years from menopause. With increasing $\mathrm{FSH}$; follicules, oocytes, embryos, implantation rates decrease, and cycle cancellation rates increase. Inspite of improvments in ART, there is no consensus on the managements of patients with poor response. Parameters like increased FSH levels, low E2 levels at hCG day (300-660 pg/ml), low oocyte samples (below 4-6), antral follicules below 3-5 at the day of hCG administration, advanced age of the patients, increased FSH dosage used, extended stimulation period are used for describe of poor ovarian response. The ESHRE meeting in Bologna at 2011 was concluded with a new consensus to describe poor overian response for a common language. i) Advanced maternal age $(>40)$ or other risk factors for POR, ii) obtaining $<=3$ oocyes with convantionel stimulation before, iii) Abnormal ovarian reserve tests (AFC $<5-7$, or $\mathrm{AMH}<0.5-1.1 \mathrm{ng} / \mathrm{ml}$ ). Again, according to the criteria of Bologna, regardless of age, a patient produces $<4$ oocytes at 2 cycles even maximal stimulation and patients aged over 40 years have poor ovarian reserve tests without using stimulation are also accepted POR. ${ }^{3}$ Many factors like diminished ovarian reserve, advanced maternal age, low levels of FSH receptor numbers, pleomorphism of FSH receptors, turner syndrome, flagyl $\mathrm{X}$ syndrome, previous radiotherapy and chemotherapy story, mutations of FMR1 gen, existence of FSH binding inhibitor at folliculer liquid, degraded signal transduction as result of FSH binding, existence of autoantibody aganist granulosa cells, deficiency of vessel web that spread of gonadotropins, low levels of GnSAF (gonadotropine surge attenuating factor) were reported for POR ethiology. And also previous surgical endometrioma, previous
PID, obesity, environmental factors, smoking and functional ovarian cysts may be counted situations may be releated POR.

\section{Clinical situation}

Number of embryo has substantial importance for IVF/ICSI success rate in POR patients. Therewith high levels of gonadotropin dosages may provide more follicule election. However, this contribution is not clear on pregnancy rates. High rates of mitochondrial DNA mutations was reported at advanced maternal age. ${ }^{5}$ And also mitochondrial DNA deletions were observed in oocytes of women at advanced ages. As a result, it is thought that decreased energy production effects mitotic activity badly. More decreased ovarian stromal blood flow is also determined in patients have POR with $3 \mathrm{D}$ power doppler. ${ }^{6}$ Also increased abortus rates were also reported in patients with poor ovarian response, as well as decreased pregnancy rates. ${ }^{7}$ In patients with POR, poor response incidance at the second cycle follows first cycle was reported as $62 \%{ }^{8}$

\section{Management of poor responders}

Although different $\mathrm{COH}$ protocols are applied for increasing IVF succes rate in these patients, there is no consesus about optimal procedure. Increasing gonadotropin dosages during $\mathrm{COH}$ procedure, using different types of gonadotropins, changing start time of gonadotropins or GnRH analogs, utilization of OCS, addition of GH, DHEA-S, CC, aromatase inhibitors, testosterone, E2, nitric oxide (L-arginine), aspirin, colony-stimulating factor, dexametasone, pyridostigmine or another adjuvants or usege of naturel cycles at Assited Reproductive Technology are recommended., ${ }^{9,10}$ Despite illegal in some countries, oocyte donation is an alternative way. But it may not always be feasible for couples because of different religious and cultural characteristics. Small number of oocyte maturation is observed as result of decresed ovarian reserve in patients with POR. Therefore acceptable pregnancy rates also have been reported if IVF/ ICSI is applied in patient's own cycles even high LH surge posibilities exist. Increasing mature follicules and embriyos is main purpose for patient with poor response. Yet there are two questions. Can we make a non-exist follicule grow? And the second one: Can we fix the quality of ruined oocyte? A succesful ART is related to especially well $\mathrm{COH}$. 
Inadequate response to gonadotropines is resulted with cancellation of cycle, decreased quality and number of embryo that will be frozen or transfer, low pregnancy rate and psychological trauma.

\section{Cycle cancellation criterias in poor respon- ders}

Similar cycle cancellation parameters are used in all IVF cycles. These are i) 3 or less follicules in USG, ii) highest $\mathrm{E} 2<500 \mathrm{pg} / \mathrm{ml}$.

\section{Clinical and laboratory administration}

Increasing the number of trial and usage of ICSI instead of IVF in patients with poor ovarian response and aged over 40 seem to provide an increase in pregnancy rates. ${ }^{11}$ Depending on decreased reserve in patients with POR a small number oocyte development is monitored. Therefore IVF-ICSI applications during naturel cycles (advantage of requiring lower drug costs) was reported with acceptable pregnancy rate in several series. ${ }^{12}$

Decreased ovarian reserve is related with decreased oocyte quality. At the end of thirties and early forties are related with increased aneuploidi rates, decreased naturel fecundity, decreased number of follicule and worsening oocyte quality. Therefore transfer of the healty oocyte cytoplasm to oocyte with a poor prognosis using microinjection procedure and transfer of the germinal vesicle of defective oocyte to denucleated healty oocyte have become a current issuse. In In vitro Maturation procedure, immature oocytes are gathered, are grown in vitro and ICSI procedure is applied. Contemporarily better follicule development is provided with this procedure. In preliminary studies of in vitro maturation (IVM), decreased cancellation of cycle and acceptable pregnancy / implantation rates were also reported. ${ }^{13}$ Assisted hatching is a newer lab technique that was developed when fertilty experts observed that embryos with a thin zona pellucida had a higher rate of implantation during IVF. Higher clinical pregnancy and implantation rates have been observed after assisted hatching. ${ }^{14}$ Increased pregnancy results with early embryo transfer in patients with POR was also reported. ${ }^{15}$

Genetic studies on embryos and transfer of elected healty embryos are named with PIG (Preimplantation Genetic). This technique is used for single gene disease. And this procedure also seems to increase IVF success in patients with POR. Endometrial co-culture environments; as also known imitation uterus; are systems allow development of embryos in nearly naturel environment before transfer period. Higher implantation and pregnancy rates are obtained with co-culture techniques. ${ }^{16}$

In recent years another technique initiated is electroacupuncture treatment. Electroacupunctre is the usage of electric impulse stimulation with acupuncture needles. Increased oocyte quality and better pregnancy outcomes for electroacupunctre treatment undergoing patients with POR have been also reported. ${ }^{17}$ And also stem cell technology will be in treatment of POR patients in the future. Studies on the use of embryonic stem cells in reproductive medicine is promise for patients that have no oocytes or sperm.

Although studies are experimental to gain differentiated gamete cells from embryonic stem cells, succesful results have been reported. With the beginning of the formation of embryonic bodies in the mouse stem cell culture, expression of the marker of germ cells were shown. When ,germ cell markers expressing cells was cultured with retinoic acid solution to obtain male germ cell, these cells is transformed to preseperm cells. Again, culturing the embryonic bodies with the solution contains neonatal testis tissue, was succesful to gain ovarian tisuue contains oocyte-like structures These ocytes like structures have been shown to express markers specific for oocytes, and the indicator for meiosis SPC 3 expression. ${ }^{18}$ Again, in vitro male gamet cell differentiation from mouse embryonic stem cell and the injection of the obtained cell to the oocyte has been successful in obtaining blastocyst formation. ${ }^{19}$ Non- tail sperm gain in mouse and fertilization was shown when injected into the oocytes was reported by other studies..$^{20}$ It was shown that $0.1 \%$ of human embryonic stem cells was differentiated to primordial germ cells by the surface and gene expression markers. ${ }^{21}$ In another study in the mouse, oocyte gain from embryonic stem cells has been successful. ${ }^{22}$ And also trofoblaste differentiation from embryonic bodies gained from embryonic stem cell and, human chorionic gonadotrophine secretion from these trofoblastes was shown. ${ }^{23}$ There are some limitations in the use of stem cells in treatment. It is known the potential of stem cells diferentiate various cells, the mechanisms of this differentiation is not certain. Before the use in the treatment all of the mechanisms in differentiation, potential side effects and in vivo situation of these cells must be illuminated. And also stem cell applications have some ethical problems. In the use of setm cells in clinical practice in the future most studies has to be made on animal experiments.

\section{Treatment of poor responders}

Assuming that ovarian reserve tests predict an acceptable success rate, the next question is what stimulation protocol would optimize patients chances for success. Contemporarily despite of technological progresses, IVF is still an expencive treatment. More gonadotropin doses are used in patients with POR and it increases costs. Prolonged treatments and high rates of cycle cancellation are another important problems in POR. The best ovarian hyperstimulation procedure in these patients should provide low cycle cancellation rates, enough number of mature oocyte, lower cost, optimal pregnancies, more lived births. However the best treatment for PORs might be discussed.

\section{Administration of gonadotropin}

The first and basic approach to the poor responder patients is seems to increase the dose of gonadotropins when inadequate response is obtained with standart dose ovarian stimulation in $\mathrm{COH}$ cycles. Decrased cycle cancellation rates and increased pregnancy rates were reported in a study that gonadotropin doses were increased from 350UI to 400UI. ${ }^{24}$ Manzi et al. ${ }^{25}$ reported that they got more oocytes with 150UI increment of daily FSH dose. Yet an increment for pregnancy rates was not reported. In another study, FSH dose was increased to 450UI cause of there was no adequte response to 250UI and more follicules were gathered. Yet pregnancy rates were observed low. ${ }^{26}$ Again another studies in patients with POR, it was observed that increasing gonadotropin dose had no effect on level of E2, number of embryo and pregnancy rates. ${ }^{27}$ In spite of increased FSH dose in patients with POR, poor oocyte retrieval may be related with poor ovarian reserve. However increasing FSH dose seems to fail about oocyte retrieval, recombinant FSH is shown more potent than urinary products for number of oocyte rerieved, more embryo obtain and higher pregnancy rates.

FSH and LH have same parts in folliculogenesis and ovulation. Barrenetxea et al. ${ }^{28}$ have reported that addition of rLH to stimulation protocol after seventh day of cycle has no effect on clinical pregnancy, implantation rates and cycle Dynamics. ${ }^{28}$ Also, a recent prospective 
randomized study demonstrated that the additional exogenous LH activity in the form of either recombinant $\mathrm{LH}$ or low-dose recombinant hCG did not improve the cycle outcomes and the pregnancy rates in poor responders. ${ }^{29}$

\section{Administration of gnrh analogues}

In recent years, the advantages of microdose GnRH agonist flareup technic have been reported in some studies. ${ }^{30}$ In several studies, GnRh antagonists have been found avail. ${ }^{31}$ In a previous study that compares two stimulation protocols, we have observed no difference between them. ${ }^{32}$

\section{GnRH agonists}

GnRH agonists are one of the main medicines of $\mathrm{COH}$ cycles. GnRH agonists supress the production of pituitary gonadotropin and become effective in preventing premature LH surge and increase gonadotropin require. Decreasing GnRH dose in patients with POR decreases gonadotropin require and increases number of oocyte. ${ }^{33}$ Existence of $\mathrm{GnRH}$ receptors in human ovarian tissue that is shown by studies indicates that agonists may have direct and negative effects on over beside pituitary. This situation have caused an argument about classical usage of $\mathrm{GnRh}$ agonist in patients have limited ovarian reserve. Therefore modified agonist protocols have been identified in patients with POR. There are many different modified GnRH-a protocols for patients who had previously shown a poor response to long luteal GnRHa protocol- mostly by altering dose and timing of administration. Among the various types of modified GnRH-a protocols, microdose flare-up is one of the most popular regimen.

Some studies support short and flare protocols against long protocols have been reported in literature. ${ }^{34,35}$ Decreased cycles cancellation, increased pregnancy rates without premature LH surge for microdose flare-up protocol, thoroughly decreased dose (40$80 \mu \mathrm{g})$, have been reported. ${ }^{36}$ In microdose GnRH agonist (GnRH-a) flare-up protocol; the ovarian suppression is not excessive and the initial stimulation of GnRH receptors and consequent secretion of endogenous gonadotropins reinforce the effects of exogenously administered gonadotropins. These are main advantages of this protocol.

In a recent rewiev, comparison of two agonist protocols, there was no statistically difference between two protocols. These protocols are stop and non-stop long GnRH agonist protocols. In the stop agonist protocol GnRH agonist was initiated in the midluteal phase and was stopped upon adequate down regulation. In the non-stop protocol, a standard long GnRH agonist was applied and GnRH agonist administration continued until the day of hCG administration. ${ }^{37}$

In a study that considers co-flare 450 cycles in POR patients retrospectively, $24 \%$ cycle cancellation, $20 \%$ pregnancy rate per cycle, $14 \%$ live birth rate have been reported. Lower cycle cancellation rate is deteceted in patients have 'Estradiol dubling'. Flare effect at the begining seems better stimulation indicator but has no effect on pregnancy outcomes significantly. ${ }^{38}$

\section{GnRH antagonists}

Defining extra-pituitary effects of GnRH and possible importance of these effects on poor ovarian responders caused to head finding different cycles seeking instead of classical long luteal agonist cycles. First times, these negative effects were tried to overcome with modified agonist procedures. With invention of GnRH antagonists, usage of these medicines in POR patients have become current issue. Most recently the use of GnRH antagonists has been suggested as the preferred ovarian stimulation protocol in poor responders. In recent days use of GnRH antagonists in patients with POR represent a new perspective to clinicians. And studies about this issue increase gradually. Lower cycles cancellation rates, more number of oocyte, more transferable number of embryo and higher clinical pregnancy rates with antagonists have been reported in studies compare GnRH antagonists with especially the usage of long luteal GnRH analogs in poor responders. ${ }^{39}$ As the reason, more excessive supression of $\mathrm{GnRH}$ analogs on overs in patients already have poor ovarian reserve has been claimed. Nevertheless FSH and LH blood serum levels are supressed excessively at the third day of cycles used GnRHa, when ovarian stimulation starts, blood serum FSH and LH levels are frequently 5-8IU at cycles used GnRH antagonists. Early LH peak is prevented with addition of $\mathrm{GnRH}$ antagonists at late folliculer period of stimulation protocol. Thus GnRH antagonist protocols, in proportion to GnRH agonist long protocols, do not supress endogenous FSH and $\mathrm{LH}$ at the early follicular period and allow natural follicule election. ${ }^{40}$

In a study was performed on 48 POR patients, equal pregnancy rates have been reported between microdose flare-up and antagonist protocols. ${ }^{41}$ Cycle cancellation rate were found higher in antagonist group in comparison to long protocol group in a study that the evaluation performed when the leading follicul was reached $16 \mathrm{~mm} .{ }^{42}$ In the study, series of Humaidan et al. ${ }^{43}$ with 72 patients, compared flexible GnRH antagonis protocols with long protocol significant diffences were detected for number of follicule, number of oocyte, implantation and pregnancy rates. ${ }^{43}$

In a study performed by Fsouliotis et al.,the stimulation was made with antagonist protocols at new cycle to 53 patients could not be pregnant with long protocol at previous cycle. Higher implantation, pregnancy rates and pregnancy ongoing rate were detected in antagonist group. Marci et al. $^{44}$ who compared ovarian response in antagonist protocol with standard long protocol, reported more number of follicule and lower cancellation rates in antagonist group. ${ }^{45}$

In a recent meta-analysis compared GnRH agonists and antagonists, better outcomes for antagonist protocols in proportion to analogs, lower cycle cancellation rate, more number of oocyte and metaphase 2 oocyte, higher clinical pregnancy rate were determined. Neverthless there was no significant differences between antagonist protocols with flare-up protocols, More number of oocytes were gathered in flare-up protocols than antagonist protocols yet. ${ }^{12}$

Despite these theoretical advantages of $\mathrm{GnRH}$ antagonists, there is some concern that the use og $\mathrm{GnRH}$ antagonists in poor responders may have adverse effects on ovarian steridogenesis, follicular growth, embryo development and implantation process. ${ }^{46}$

In the study of Di Luigi et al. ${ }^{47}$ that compared microdose leuprolid asetat protocol with protocols that got started with GnRH antagonists and $\mathrm{E} 2$ replacement at luteal phase, no significant is detected for cycle cancellation, number of oocyte, clinical pregnancy rates between these two groups. ${ }^{47}$

In a study including 300 consecutive cycles, similar pregnancy rates are found between the microdose flare-up group and antagonist. ${ }^{48}$

\section{Aromatase inhibitors}

Aromatase inhibitors inhibit last step of estradiol synthesis. Letrozole the 3. generation selective aromatase inhibitor firstly used 
to decrease gonadotropin dose at ovulation induction. It was shown that aromatase inhibitors have positive effects on follicular response aganist FSH in poor responder patients. ${ }^{49}$ In our earlier study including 70 patients undergoing flexible antagonist protocol, it was shown that adjunctive letrozole administration seems to restore an IVF cycle by decreasing the rate of cycle cancellation and seems to reduce the cost by reducing the total gonadotropin dosage. ${ }^{50}$

In a study including 12 POR patients, less gonadotropin require was detected with $2.5 \mathrm{mg}$ (7 days) letrozole. ${ }^{51}$ More over in another study, 71 of 147 patients had cancelled cycle, got high dosage of FSH/ $\mathrm{hMG}+$ antagonist and $2.5 \mathrm{mg}$ Letrozole, and when this group outcomes were compared with the group got same protocol without letrozole addition, increased testosterone in follicular liquid, androstenedion concantrations, number of oocytes gathered and implantation rates were detected significantly higher in letrozole group. ${ }^{52}$

\section{Additional treatments}

Passage of egg cells to functional pool that is sensitive to reproductive hormone, take about four months. In present day, modern medicine has drugs and knowledges can interfere in last 15-20 days of this process. There are some hormones detected; that are believed to effect earlier periods of this process.

\section{DHEA (dehydroepiandrosterone)}

Dehydroepiandrosterone is an endogenous steroid that originates from zona reticularis (\%80) of the adrenal cortex and from ovarian theca cells $(\% 20)$. Dehydroepiandrosterone is an essentian prohormone in ovarian follicular steroidogenesis. The level is decreased with age. This hormone converts to estrogen and androgen in women. This convertion is in favor of androgens. In case reports and laboratuary studies, It was shown that test-tube baby and fertilization administrations of DHEA increase pregnancy rates and live births rates in patients with poor ovarian reserve, advanced ages $(>38) .{ }^{53}$ At the same time it was shown that DHEA decreases abortion rates. In our earlier study, It is shown that addition of DHEA provides enhancement on IVF outcomes. ${ }^{54}$ There is thought about that DHEA increases number oocytes passed functional reserve and decreases aneuploidi by opitumum effect on oocyte cytoplasm. ${ }^{55}$ Therefore $6-8$ weeks administrations of DHEA ( $75 \mathrm{mg} /$ gün) have become a routine in POR patients contemproraliy.

\section{GH (Growth hormone)}

It is known that growth hormone has a benefical effect on ovarian function. In laboratuary studies, it is shown that GH provides to increase oocyte maturation and capacity of DNA repair in human cells. In a study including over 40 aged women, addition of GH to treatment augmented pregnancy and live births rates significantly. ${ }^{56}$ Again in another study, positive outcomes were reported. ${ }^{57}$ Usage of $\mathrm{GH}$ has become a routine for treatment protocols in patients have no response..$^{58}$ But addition of GH releasing factor in poor responders undergoing IVF treatment does not appear to beneficial.

\section{Metformin}

Today, the use of metformin is not offered to treat anovulatory infertil women. In patients with reduced ovarian reserve, metformin worsens the response to gonadotropins. It is thought that the use of metformin reduces response to stimulatin by reducing androgen levels. ${ }^{59}$

\section{COC pre-treatment}

COC pre-treatment supresses endogenous gonadotropins and sensitizes estrogen receptors depending on estrogen components inside. Coperman et al. reported increased pregnancy rate, and decreased cancellation rate in the antagonist cycles of poor responder patients who received OCP treatment, when compared with patients not receiving OC pills. ${ }^{40}$

\section{Androgen}

Androgens play a critical role on follicular growth. Androgens receptors have been identified in the human ovary. The addition of androgen during the early follicular phase might have a beneficial effect on the number of small antral follicles and improve the ovarian sensitivity to FSH. In a meta-analysis, it was detected that the use of transdermal testosterone in POR patients has benefits for live birth rates, clinical pregnancies per cycle and gonadotropin doses used in group received testosterone significantly. ${ }^{60}$

\section{Luteal phase supplementation}

Luteal supplementation with either $\mathrm{hCG}$ or progesterone significantly improves fertility outcomes compared with no treatment. Addition of oral estrogen to progesterone also improves implantation rates. So luteal phase support is mostly offered in IVF/ICSI protocols. But it has a potential risk of OHSS. ${ }^{61}$

\section{Expert commentary}

Poor response criterias are increaed cycle cancellation rates, poor embryo development, decreased pregnancy rates in POR patients. Assessment of ovarian reserve before $\mathrm{COH}$ is important for the choise of adequate protocol. Various solution strategies have been tried for increasing IVF success in POR patients. Some variations have been made for type, dose and timing of gonadotropins, agonists and antagonists, a certain superiority could not have been shown between them yet. Addition of adjuvants to treatmant has contributed to IVF success. Individualising $\mathrm{COH}$ protocols for each patient seems like more appropriate.

\section{5-year view}

Main factor that effects fertility success ,as a result of ovarian aging effect, is defect of oocyte quality. A lot of stimulation protocol have been offered for increasing quality and number of oocyte. Importance of adjuvants and hormones supports seem like become more important with devoloping technology and increasing knowledges. Developments in IVF technology increases day by day. Electroacupunctre is one of these. In recent years, increasing stem cell studies give hope. In future maybe we can retrieve oocytes from stem cells.

\section{Financial \& competing interests disclosure}

The author has no relevant affiliations or financial involvement with any organization or entity with a financial interest in or financial conflict with the subject matter or materials discussed in the manuscript. This includes employment, consultancies, honoraria, stock ownership or options, expert testimony, grants or patents received or pending, or royalties. No writing assistance was utilized in the production of this manuscript. 


\section{Key issues}

i. Short protocol has flareup effect on pituitary gonadotropin release, on the other hand the use of long protocol results more coordinated follicular growth.

ii. Use of GnRH antagonists has better results regarding stimulation time, gonadotropine total dosage, and retrieval of oocytes. But more comperative studies are required.

iii. There are no differnces between the long GnRH agonist protocols and $\mathrm{CC}+\mathrm{rFSH}$ in $\mathrm{GnRH}$ antagonist protocols.

iv. GnRH antagonist and short GnRH agonist protocols seems similar regarding the pregnancy rates.

v. Natural cycle IVF can be an alternative to standard ovarian stimulation. Natural cycle is less invasive, and less costly. This can be offered for poor responders who do not produce more oocytes with ovarian stimulation.

vi. Short GnRH and long GnRH agonist protocols have no differences.

vii. Recombinant FSH is shown more potent than urinary products for number of oocytes retrieved, more embryo obtain, and higher pregnancy rates in IVF protocols.

viii. Shortening the duration of embryo culture might be associated with an improvement in pregnancy rates. So early embryo transfer in POR might be beneficial.

ix. Adjuvant treatment allows an increase in the success of IVF. Especially addition of GH appears to improve the probabilty of pregnancy.

\section{Acknowledgements}

None.

\section{Conflict of interest}

The author delcares no conflict of interets.

\section{References}

1. J Garcia, GS Jones, AA Acosta, et al. Corpus luteum function after follicle aspiration for oocyte retrieval. Fertil Steril. 1981;36(5):565-572.

2. Elassar A, Engmann L, Nulsen J, et al. Letrozole and gonadotropins versus luteal estradiol and gonadotropin-releasing hormone antagonist protocol in women with a prior low response to ovarian stimulation. Fertil Steril. 2011;95(7):2330-2334.

3. Ferraretti AP, La Marca A, Fauser BC, et al. ESHRE consensus on the definition of 'poor response' to ovarian stimulation for in vitro fertilization: the Bologna criteria. Hum Reprod. 2011;26(7):1616-1624.

4. Martinez F, Barri PN, Coroleu B, et al. Women with poor response to IVF have lowered circulating gonadotrophin surge-attenuating factor (GnSAF) bioactivity during spontaneous and stimulated cycles. Hum Reprod. 2002;17(3):634-640.

5. Barritt JA, Cohen J, Brenner CA. Mitochondrial DNA point mutation in human oocytes is associated with maternal age. Reprod Biomed Online. 2000;1(3):96-100.

6. Pan HA, Wu MH, Cheng YC, et al. Quantification of ovarian stromal Doppler signals in poor responders undergoing in vitro fertilization with three-dimensional power Doppler ultrasonography. Am J Obstet Gynecol. 2004;190(2):338-344.
7. Levi AJ, Drews MR, Bergh PA, et al. Controlled ovarian hyper stimulation does not adversely affect endometrial receptivity in in vitro fertilization cycles. Fertil Steril. 2001;76(4):670-674.

8. Klinkert ER, Broekmans FJ, Looman CW, et al. A poor response in the first in vitro fertilization cycle is not necessarily related to a poor prognosis in subsequent cycles. Fertil Steril. 2004;81(5):1247-1253.

9. Frydman R. Poor responders: still a problem. Fertil Steril. 2011;96(5):1057.

10. Shanbhag S, Aucott L, Bhattacharya S, et al. Interventions for 'poor responders' to controlled ovarian hyper stimulation $(\mathrm{COH})$ in in-vitro fertilisation (IVF). Cochrane Database Syst Rev. 2007;24(1):CD004379.

11. Maman E, Geva LL, Yerushalmi G, et al. ICSI increases ongoing pregnancy rates in patients with poor response cycle: multivariate analysis of 2819 cycles. Reprod Biomed Online. 2012;25(6):635-641.

12. Franco JG, Baruffi RL, Mauri AL, et al. GnRH agonist versus GnRH antagonist in poor ovarian responders: a meta-analysis. Reprod Biomed Online. 2006;13(5):618-627.

13. Liu J, Lu G, Qian Y, et al. Pregnancies and births achieved from in vitro matured oocytes retrieved from poor responders undergoing stimulation in in vitro fertilization cycles. Fertil Steril. 2003;80(2):447-449.

14. Practice Committee of Society for Assisted Reproductive Technology, Practice Committee of American Society for Reproductive Medicine. The role of assisted hatching in in vitro fertilization: a review of the literature. A Committee opinion. Fertil Steril. 2008;90(5):196-198.

15. Bahceci M, Ulug U, Ciray HN, et al. Efficiency of changing the embryo transfer time from day 3 to day 2 among women with poor ovarian response: A prospective randomized trial. Fertil Steril. 2006;86(1):81-85.

16. Rubio C, Simon C, Mercader A, et al. Clinical experience employing coculture of human embryos with autologous human endometrial epithelial cells. Hum Reprod. 2000;15(6):31-38.

17. Zheng $\mathrm{CH}$, Zhang MM, Huang GY, et al. The role of acupuncture in assisted reproductive technology. Evid Based Complement Alternat Med. 2012;2012:543924.

18. Lacham-Kaplan O, Chy H, Trounson A. Testicular cell conditioned medium supports differentiation of embryonic stem cells into ovarian structures containing oocytes. Stem Cells. 2006;24(2):266-273.

19. Geijsen N, Daley GO. Male germ cells. Methods Enzymol. 2006;418:307314.

20. Daley GO. Customized human embryonic stem cells. Nat Biotechnol. 2005;23(7):826-828.

21. Aflatoonian B, Moore H. Germ cells from Mouse and human embryonic stem cells. Reproduction. 2006;132(5):699-707.

22. Hubner K, Fuhrmann G, Christenson LK. Derivation of oocytes from mouse embryonic stem cells. Science. 2003;300(5623):1251-1256.

23. Golos TG, Pollastrini LM, Gerami-Naini B. Human embryonic stem cells as a model for trophoblast differantiation. Semin Reprod Med. 2006;24(5):314-321.

24. Hofmann GE, Toner JP, Muasher SJ, et al. High-dose follicle-stimulating hormone (FSH) ovarian stimulation in low-responder patients for in vitro fertilization. J In Vitro Fert Embryo Transf. 1989;6(5):285-289.

25. Manzi DL, Thornton KL, Scott LB, et al. The value of increasing the dose of human menopausal gonadotropins in women who initially demonstrate a poor response. Fertil Steril. 1994;62(2):251-256.

26. Land JA, Yarmolinskaya MI, Dumoulin JC, et al. High-dose human menopausal gonadotropin stimulation in poor responders does not improve in vitro fertilization outcome. Fertil Steril. 1996;65(5):961-965. 
27. Schoolcraft W, Schlenker T, Gee M, et al. Improved controlled ovarian hyper stimulation in poor responder in vitro fertilization patients with a microdose follicle-stimulating hormone flare, growth hormone protocol. Fertil Steril. 1997;67(1):93-97.

28. Barrenetxea G, Agirregoikoa JA, Jime'nez MR, et al. Ovarian response and pregnancy outcome in poor-responder women: a randomized controlled trial on the effect of luteinizing hormone supplementation on in vitro fertilization cycles. Fertil Steril. 2008;89(3):546-553.

29. Berkkanoglu M, Isikoglu M, Aydin D, et al. Clinical effects of ovulation induction with recombinant follicle-stimulating hormone supplemented with recombinant luteinizing hormone or low-dose recombinant human chorionic gonadotropin in the midfollicular phase in microdose cycles in poor responders. Fertil Steril. 2007;88(3):665-669.

30. Demirol A, Gurgan T. Comparison of microdose flare-up and antagonist multiple-dose protocols for poor-responder patients: a randomized study. Fertil Steril. 2009;92(2):481-485.

31. Cheung LP, Lam PM, Lok IH, et al. GnRH antagonist versus long GnRH agonist protocol in poor responders undergoing IVF: a randomized controlled trial. Hum Reprod. 2005;20(3):616-621.

32. Kahraman K, Berker B, Atabekoglu CS, et al. Microdose gonadotropin-releasing hormone agonist flare-up protocol versus multiple dose gonadotropin-releasing hormone antagonist protocol in poor responders undergoing intracytoplasmic sperm injection-embryo transfer cycle. Fertil Steril. 2009;91(6):2437-2444.

33. Olivennes F, Schneider Z, Remy V, et al. Perinatal outcome and followup of 82 children aged 1-9 years old conceived from cryopreserved embryos. Hum Reprod. 1996;11(7):1565-1568.

34. Tarlatzis BC, Zepiridis L, Grimbizis G, et al. Clinical management of low ovarian response to stimulation for IVF: a systematic review. Hum Reprod Update. 2003;9(1):61-76.

35. Detti L, Williams DB, Robins JC, et al. A comparison of three downregulation approaches for poor responders undergoing in vitro fertilization. Fertil Steril. 2005;84(5):1401-1405.

36. Surrey ES, Bower J, Hill DM, et al. Clinical and endocrine effects of a microdose GnRH agonist flare regimen administered to poor responders who are undergoing in vitro fertilization. Fertil Steril. 1998;69(3):419424 .

37. Kyrou D, Kolibianakis EM, Venetis CA, et al. How to improve the probability of pregnancy in poor responders undergoing in vitro fertilization: a systematic review and meta-analysis. Fertil Steril. 2009;91(3):749-766.

38. Spandorfer S, Navarro J, Kump LM, et al. "Co-Flare" stimulation in the poor responder patient: predictive value of the flare response. $J$ Assist Reprod Genet. 2001;18(12):629-633.

39. Cheung LP, Lam PM, Lok IH, et al. GnRH antagonist versus long GnRH agonist protocol in poor responders undergoing IVF: a randomized controlled trial. Hum Reprod. 2005;20(3):616-621.

40. Copperman AB. Antagonists in poor-responder patients. Fertil Steril 2003;80(1):16-24.

41. Akman MA, Erden HF, Tosun SB, et al. Comparison of agonistic flareup-protocol and antagonistic multiple dose protocol in ovarian stimulation of poor responders: results of a prospective randomized trial. Hum Reprod. 2001;16(5):868-870.

42. D’Amato G, Caroppo E, Pasquadibisceglie A, et al. A novel protoco of ovulation induction with delayed gonadotropin-releasing hormone antagonist administration combined with high-dose recombinant follicle-stimulating hormone and clomiphene citrate for poor responders and women over 35 years. Fertil Steril. 2004;81(6):1572-1577.

43. Humaidan $\mathrm{P}$, Bungum L, Bungum M, et al. Reproductive outcome using a GnRH antagonist (cetrorelix) for luteolysis and follicular synchronization in poor responder IVF/ICSI patients treated with a flexible GnRH antagonist protocol. Reprod Biomed Online. 2005;11(6):679-684.
44. Fasouliotis SJ, Laufer N, Sabbagh-Ehrlich S, et al. Gonadotropin-releasing hormone $(\mathrm{GnRH})$-antagonist versus $\mathrm{GnRH}$-agonist in ovarian stimulation of poor responders undergoing IVF. J Assist Reprod Genet. 2003;20(11):455-460

45. Marci R, Caserta D, Dolo V, et al. GnRH antagonist in IVF poor-responder patients: results of a randomized trial. Reprod Biomed Online. 2005;11(2):189-193.

46. Hernandez ER. The clinical benefits of recombinant gonadotrophins. Hum Reprod. 2000;15(3):734-745.

47. DiLuigi AJ, Engmann L, Siano LJ, et al. Leuprolide Acetate trigger effectively prevents OHSS without compromising clinical outcomes in patients with serum estradiol $>4,000 \mathrm{pg} / \mathrm{ml}$ in IVF cycles. Fertil Steril. 2007;88(1):118.

48. Ulug U, Ben-Shlomo I, Turan E, et al. Conception rates following assisted reproduction in poor responder patients: a retrospective study in 300 consecutive cycles. Reprod Biomed Online. 2003;6(4):439-443.

49. Mitwally MF, Casper RF. Aromatase inhibition improves ovarian response to follicle-stimulating hormone in poor responders. Fertil Steril. 2002;77(4):776-780.

50. Ozmen B, Sönmezer M, Atabekoglu CS, et al. Use of aromatase inhibitors in poor-responder patients receiving $\mathrm{GnRH}$ antagonist protocols. Reprod Biomed Online. 2009;19(4):478-485.

51. Mitwally MFM, Casper RF. Aromatase inhibition reduces the need for gonadotropins in controlled ovarian hyperstimulation. J Soc Gynecol Investig. 2004;11(6):406-415.

52. Garcia-Velasco JA, Moreno L, Pacheco A, et al. The aromatase inhibitor letrozole increases the concentration of intraovarian androgens and improves in vitro fertilization outcome in low responder patients: a pilot study. Fertil Steril. 2005;84(1):82-87.

53. Wiser A, Gonen O, Ghetler Y, et al. Addition of dehydroepiandrosterone (DHEA) for poor-responder patients before and during IVF treatment improves the pregnancy rate: a randomized prospective study. Hum Reprod. 2010;25(10):2496-2500.

54. Sönmezer M, Ozmen B, Cil AP, et al. Dehydroepiandrosterone supplementation improves ovarian response and cycle outcome in poor responders. Reprod Biomed Online. 2009;19(4):508-513.

55. Gleicher N, Weghofer A, Barad DH. The role of androgens in follicle maturation and ovulation induction: friend or foe of infertility treatment? Reprod Biol Endocrinol. 2011;9:116 p.

56. Tesarik J, Hazout A, Mendoza C. Improvement of delivery and live birth rates after ICSI in women aged $>40$ years by ovarian co-stimulation with growth hormone. Hum Reprod. 2005;20(9):2536-2541.

57. Kucuk T, Kozinoglu H, Kaba A. Growth hormone co-treatment within a GnRH agonist long protocol in patients with poor ovarian response: a prospective, randomized, clinical trial. J Assist Reprod Genet. 2008;25(4):123-127.

58. Yovich JL, Stanger JD. Growth hormone supplementation improves implantation and pregnancy productivity rates for poor-prognosis patients undertaking IVF. Reprod Biomed Online. 2010;21(1):37-49.

59. Palomba S, Falbo A, Di Cello A, et al. Does metformin affect the ovarian response to gonadotropins for in vitro fertilization treatment in patients with polycystic ovary syndrome and reduced ovarian reserve? A randomized controlled trial. Fertil Steril. 2011;96(5):1128-1133.

60. González-Comadran M, Durán M, Solà I, et al. Effects of transdermal testosterone in poor responders undergoing IVF: systematic review and meta-analysis. Reprod Biomed Online. 2012;25(5):450-459.

61. Pritts EA, Atwood AK. Luteal phase support in infertility treatment: a meta-analysis of the randomized trials. Hum Reprod. 2002;17(9):22872299 . 